\title{
A Review on Hydrodynamical Stability of Thin Film Flowing Along an Inclined Plane
}

\author{
Souradip Chattopadhyay $^{1^{*}}$, Anandamoy Mukhopadhyay ${ }^{2}$ and Amlan K. Barua ${ }^{1}$ \\ ${ }^{1}$ Department of Mathematics, Indian Institute of Technology Dharwad, Karnataka - 580011, India \\ ${ }^{2}$ Department of Mathematics, Vivekananda Mahavidyalaya (The University of Burdwan), West Bengal - 713103, India \\ ${ }^{*}$ Corresponding author
}

\begin{abstract}
Article Info
Keywords: Hydrodynamical stability, Inclined plane, Thin film.

2010 AMS: 35Q30, 35Q35, 35Q79,

76E07, 76E17, 76E25, 76E30.

Received: 9 September 2018

Accepted: 8 April 2019

Available online: 30 August 2019
\end{abstract}

\begin{abstract}
The dynamics and stability of thin liquid films have fascinated scientists over many decades. Thin film flows occur over a wide range of length scales and are central to numerous areas of engineering, geophysics and biophysics. These include nanofluidics and microfluidics, lava flows, coating flows, tear-film rupture, dynamics of continental ice sheets and surfactant replacement therapy. Study of falling film instability has its wide applications in the practical field of industry and engineering. Practical applications in industrial processing motivate the recent research to investigate the factors which may affect the formation of waves on the surface of the coating layers and/or to determine the ways to overcome or to minimize the unwanted factors within the desired limit of tolerance. The dynamics of a liquid film flowing down a plane under the action of gravity is a problem which appears in many technological and natural systems, namely large scale geophysical environments such as lava flows or spillways, daily life scenarios such as water flowing down a window pane or a slippery road on a rainy day, chemical engineering processes such as evaporators, heat exchanges and falling film reactors or surface coating. The aim of this paper is to throw light on the studies conducted on hydrodynamical stability.
\end{abstract}

\section{Introduction}

Hydrodynamical stability is an important branch of the fluid mechanics both from application and theoretical points of view. Stability is the measure of the ability of a system to resist the changes or in other words how a system reverts to its original state after suffering all possible modes of perturbation. An important criterion for a flow state to be physically stable is the ability of the system to withstand small disturbances which are present or may be transmitted from the surroundings to the flow. The theory of small oscillations is a useful tool for investigating the stability problems in hydrodynamics. If small disturbances superimposed on initial laminar flow, decay in time or with distance downstream, then the laminar flow is stable. If they increase, that is if the growth of the perturbation is unbounded, or if the real outcome is just another different state, or if it is a bounded but turbulent or chaotic state or accompanies the collapse of physical assumptions underlying the governing equations then the outcome is a different physical system. A very common example of instability is the formation of waves on surfaces of water owing to the action of wind. The "Taylor-Couette flow instability" is a well known instability, which arises due to centrifugal force, and Rayleigh-Benard convection, which arises due to difference of density are important instability phenomena occurring in laboratories and nature. A detailed description of the entire field is given by Schlichting [1], Lin [2], Hsieh and Ho [3] and Drazin and Reid [4]. It is very interesting that despite the effort of generations of applied mathematicians, beginning with Rayleigh, Kelvin and Reynolds, many simple understanding of phenomena related to hydrodynamic stability remain incomplete. Therefore it is an important task for the applied mathematics community to highlight different aspects of flow instability.

The instability of a thin film was first investigated in 1949 when Kapitza and Kapitza [5] took some elegant photographs with the help of some simple kitchen apparatus. After the publication of these photographs, the research community felt that these photographs represent a simple hydrodynamical instability phenomenon on a thin film flowing along an inclined plane. The beauty and simplicity of this phenomenon

Email addresses and ORCID numbers: 183111001@iitdh.ac.in, 0000-0002-4418-6201 (S. Chattopadhyay), ananda235@email.com (A. Mukhopadhyay), abarua@iitdh.ac.in (A. K. Barua) 
lie in the fact that although the movement of the film is due to inertia, the instability may occur at an extremely low Reynolds number under very common conditions. Their simplicity also lies in the fact that the wavy thin film flowing down an inclined plane can be parameterized by a single dimensionless parameter (Reynolds number) with mild approximation. For example, a simple tap water flow is sufficient to produce a wavy thin film. When the work of Kapitza and Kapitza [5] came to light, many top applied mathematicians became attracted to know the instability mechanism of the wavy thin film due to its added complexity of the involved free surface. The factors behind the origin of instability was first understood by Benjamin [6] in Cambridge through the use of long-wave expansion method. At Michigan, C. S. Yih [7] improved the theory of Benjamin by introducing the vital role of surface tension on a thin film. D. J. Benney [8] at MIT pioneered a lubrication approach to include nonlinear effects. Later Shkadov [9, 10] of Moscow State University introduced the averaging technique and then Sivashinsky [11] in Russia derived the famous Kuramoto-Sivashinsky equation for the falling film instability. Benjamin [6] and Yih [7] developed linear theories which were only able to describe the instability of the flat film in basic state. On the other hand the nonlinear theories developed by Benney [8], Shkadov [9, 10] and Sivashinsky [11] helped to describe only the wave dynamics of first 10 cm of the inclined plane but failed to demystify the rest $1 \mathrm{~m}$ of the inclined plane. In the eighties, the theory of instability of falling film was improved significantly by the use of computational methods, which allows the simulation of wave dynamics within $1 \mathrm{~m}$ channel to fully capture the entire sequence of the wave evolution. Pumir et al. [12] pointed out that the complex interfacial dynamics of a falling film often consist of quasi-stationary travelling waves which propagate long distances without changing their shape or speed. Theoretical as well as numerical investigations are still going on to explore the complex wave dynamics of a wavy thin film.

\section{Review of Literature}

Hydrodynamic mode of instability on a surface of an isothermal falling film was first shown by Kapitza and Kapitza [5] in their experimental work. After this experimental work, a thin viscous film flowing along a vertical/inclined wall became the topic of highest interest for many researchers. Benjamin [6] and Yih [7] studied the isothermal falling film by the way of linear stability analysis and predicted that when the Reynolds number $R e$, which is a flow parameter, exceeds some critical value $R e_{c}=\frac{5}{6} \cot \theta$ (where $\theta$ was the angle of inclination with the horizon), the uniform flow becomes unstable to long-wave disturbances. Benney's [8] long-wave model has considered the evolution of the finite-amplitude disturbances of the flat film. In this model the velocity components and pressure gradient are expanded in powers of $\varepsilon$, which is the ratio of the thickness of the flat film to the wave length of the disturbances in the direction of the flow. Under the assumption of long-wave length $(\varepsilon<<1)$ the governing equations together with the boundary conditions reduced to a single nonlinear partial differential equation, that describes the evolution of the free surface. Later, a nonlinear analysis was performed by Gjevik [13], Lin [14], Nakaya [15] and others. Lin [16] and Gjevik [13] found that the supercritical stability was possible in the region near the upper branch of the neutral stability curve. Nakaya [15] showed that in the presence of surface tension, an initially growing monochromatic wave reaches an equilibrium state of finite amplitude. Lin and Krishna [17] extended the analysis of Lin [16] for investigating the nonlinear stability of a liquid film with respect to the three dimensional side-band disturbances. They showed that the finite amplitude, supercritical stable, long monochromatic wave is stable to three-dimensional side-band disturbances under the modal interaction if the bandwidth is less in magnitude than $\varepsilon$, which is proportional to the ratio of the amplitude to the film thickness. The equations derived by Gjevik [13] was solved numerically by Pumir et al. [12] and they obtained its solitary wave solutions, which were earlier observed by Kapitza, Paul, Liu and Gullub [18]. Further, Liu and Gullub $[19,20]$ studied the flow development down a plane inclined at small angles $\left(4^{\circ} \leq \theta \leq 10^{\circ}\right)$ for moderate Reynolds $(6<R e<26)$ and Weber numbers $(3<W e<33)$, by introducing artificial perturbations of varying amplitudes and frequencies at the entrance, so that the two-dimensional regular waves appeared slightly downstream. They confirmed with careful measurements the theoretical predictions for the critical Reynolds number that was earlier proposed by Benjamin [6] and Yih [7]. Alekseenko et al. [21], Chang [22], Fulford [23], Lin and Wang [24], Hanratty [25, 26], Hewitt and Hall-Taylor [27] and Nakaroyakov et al. [28] studied the instability mechanism of thin film and their observations were documented in books/monographs. In our careful observation we have noticed that most of the studies dealt with the flow of a thin liquid film on an inclined plane assuming that the fluid is isothermal but in practical field it is sometimes necessary to consider the thin film to be non-isothermal.

Heat is the energy transferred between one system and its environment because of the temperature difference which exists between them. In fluid medium, a bulk amount of heat is transferred by means of convection, from the higher temperature region to the lower temperature region. The temperature of the part of the fluid increases which is in contact with the hot object and that fluid expands and naturally becomes less dense. Since the expanded fluid is lighter than the surrounding cooler fluid and more dense, buoyant force causes it to rise. From the surrounding region some of the cooler fluid then flows to take the place of the rising warmer fluid and the process continues. In the case of the thin heated films, buoyancy effects are so small that it can be treated as negligible. Uniform or non-uniform heating of the thin layers may cause considerable temperature difference at the interface and thus the thermocapillary force will draw liquid from the warmer region to the cooler region. Thermocapillary or Marangoni instability is known to be one of the most ubiquitous types of instability of the heated liquid films. This arises due to the variation of the interfacial temperature along the film interface leading thus to the non-uniformity of the surface tension, which generates an interfacial shear stress. Such a stress entails, by means of viscosity, the liquid into motion, while even hotter liquid reaches the film surface and accelerates the film flow.

Pearson [29] first investigated the thermocapillary or Marangoni instability in the liquid layers. He discussed the short wave thermocapillary instability considering a horizontal liquid layer with non-deformable free surface. He found that the surface tension variation in the absence of buoyancy can lead to convection in the thin film. Later Scriven and Sternling [30] concluded that disturbances with zero wave number are always unstable by considering the surface deformation, without taking into account the force of gravity. Further the long wave thermocapillary instability was studied by Smith [31], where the force of gravity was taken into account. The combined effect of the thermocapillary instability and a number of physical factors such as condensation/evaporation, vapor recoil and rupture due to long-range attractive Van der Waals interactions have been considered by a number of authors, such as Bankoff [32] and Burelbach et al. [33] and others. Flow of a viscous film down a heated inclined/vertical plane is an interesting problem, since the presence of the thermocapilarity, couples the hydrodynamic and the thermal boundary layer equations. Goussis and Kelly [34] first studied the thermocapillary instability in a liquid film falling down an inclined uniformly heated plane based on Orr-Sommerfeld model and the linearized energy equations. They found that a heated wall has a destabilizing effect on the free surface but a cooled wall stabilizes the flow. The long wave instability of a uniform film of volatile viscous liquid falling down a uniformly heated inclined plane was investigated by Joo et al. [35] for two-dimensional disturbances. 
They took into account the thermocapillary, surface tension and evaporation effects. Actually that work was an effective extension of the Benny's [8] long wave expansion for non-isothermal film flow. For this reason the evolution equation in terms of $h(x, t)$ is known as 'Benny equation with Marangoni effect' in the literature. The terms depending on the Marangoni number are of second order in comparison with those due to gravity and mean flow. They applied the linear and nonlinear stability theory to analyse the comparison among the different types of instabilities and finally obtained similar results as that of Goussis and Kelley [34]. Later the steady-state profile for a thin, non-isothermal coating film down an inclined plane of constant temperature, was discussed by Lopez et al. [36]. They studied the linear stability of the film to spanwise disturbances and then numerically solved the steady-state and the linear disturbance equation. They found that the small heating causes a destabilizing effect in the leading front of a coating film and also found that increasing the Biot number and allowing more heat to dissipate at the liquid-gas interface inhibit the effect of the heating.

Kabov et al. [37], Kabov [38] and Scheid et al. [39] reported various experimental works on the thermocapillary/ Marangoni instability of a thin liquid film heated from below with a local heat source. In these experiments a vertically falling thin liquid film is heated from the wall side by a device. The transverse dimension of the heater is much longer than its width in the streamwise direction. The heater generates a temperature distribution at the film surface which in turn sets up surface tension gradients which drive the fluid away from the heated region. This thermocapillary flow results in the formation of a bump in the streamwise direction, the height of which increases as the heat flux supplied by the heater increases. For a certain critical value of the heat flux, an instability in the transverse direction develops. Here this instability takes the form of rivulets at the downstream edge of the bump.

Motivated by these experimental works, Kalliadasis et al. [40] studied the thermocapillary instability of a falling liquid film heated from below by a heating device on the substrate, where the transverse dimension of the heater is much longer than width in the streamwise direction as considered by Kabov [38], Kabov et al. [37] and Scheid et al. [39] in their experimental works. They found that in the region where the wall temperature gradient is positive, the free surface develops a bump and the height of this bump was found to be an increasing function of the Marangoni number. Further, they also showed that the temperature distribution on the free surface and demonstrated that for the finite Peclet number, the temperature field is distributed downstream resulting in a free-surface temperature distribution with a maximum in the region where the wall temperature gradient is negative. Later Kalliadasis et al. [41] studied the thermocapillary instability of a viscous liquid film flowing down a uniform heated plane by using integral boundary layer approach, which was introduced by Shkadov $[9,10]$ in two dimensions and Demekhin and Shkadov [42] in three dimensions for non-isothermal flows. It combines the boundary-layer approximation of Navier-Stokes equation assuming a self-similar velocity profile on the interface with the Karman-Pohlhausen averaging method in the boundary-layer theory. Kalliadasis et al. [41] assumed a parabolic velocity profile and a linear temperature profile for the transient and non-uniform flow to apply this method. Ruyer-Quil et al. [43] studied the problem by higher order weighted residual approach with polynomial expansions for both velocity and temperature field to overcome these limitations, which introduce the second order dissipative effects that are known to determine the amplitude of the front-running capillary waves. Consequently this model is fully compatible with the Benny's expansion method up to the second order. In the study above they have shown clearly that the transport of the heat by the flow plays a double role and for the small amplitude waves it stabilizes the flow but for the large amplitude waves it promotes the instability. Trevelyan et al. [44] determined an expression of the critical Reynolds number for a thin liquid film falling down a uniformly heated wall. For that purpose they have derived a free surface evolution equation under the assumption of the small Reynolds and Marangoni numbers [O(1)], to include the convective heat transport effect at a lower relevant order. They assumed that the Peclet number is large $\left(O\left(\varepsilon^{-n}\right)\right)$ for $\left.0<n<1\right)$, but in that case in the cross-stream direction, the assumption of small temperature gradient is violated. On the other hand, the experimental findings by Kabov [38], Kabov et al. [37] and Scheid et al. [39] prominently indicate that there is a certain critical value of the heat flux for the onset of instability. To achieve such a critical value, a large temperature difference (large Marangoni number) would be required as pointed out by Kalliadasis et al. [41] and the agreement becomes much better for a large temperature difference.

The long-wave theory was used by Miladinova et al. [45] to conduct a weakly nonlinear and a fully nonlinear analysis of the evolution equation governing the film thickness in the presence of thermocapillarity arising from a linear base temperature profile. They discovered that a positive base temperature gradient destabilizes the flow and exerts an influence on the amplitude and the phase speed of the finite-amplitude waves. By using a long-wave evolution equation, Miladinova et al. [46] studied the three-dimensional instabilities of nearly sinusoidal waves on vertically falling and nonuniformly heated films. Further the linear stability of finite-amplitude two-dimensional waves was analysed using the Floquet theory. Mukhopadhyay and Mukhopadhyay [47] studied the nonlinear stabilities of viscous film flows down an inclined plane with linear temperature variation. They found that the unconditional stable zone vanishes after a cut off Marangoni number whereas subcritical, supercritical and explosive zones increase with the increase in Marangoni number.

Long-wave thermocapillary instability for the case of a horizontal thin liquid layer lying upon a solid substrate for both uniform and non-uniform base temperatures was studied by Yeo et al. [48]. They found that for a non-uniform base temperature, the film height thickens near the region where temperature gradients are negligible and severely thins upstream. Demekhin et al. [49] later investigated the linear stability of a thin liquid layer falling down an inclined wall heated by a downstream, linearly increasing temperature distribution. They saw that, in the case of linearly increasing temperature distribution, thermocapillary forces can either destabilize or stabilize the flow which are in contrast to the case of uniform heating, where the thermocapillary forces have only a destabilizing effect on all instability modes.

The Benney equation with Marangoni effect has been extensively studied by many authors. Scheid et al. [50] examined the validity domain of the Benney equation with Marangoni effect and they found that thermocapillary can strongly reduce the validity domain of the Benney equation. They also ensured that the Benney equation still remains very helpful to study the thin film flows with various physical effects since it allows to describe the film evolution by one single equation with various relevant parameters. The linear and nonlinear regimes of the two long-wave instability modes for a film flowing down a uniformly heated plane by using the regularized reduced model was analyzed by Scheid et al. [51] and there they also scrutinized the influence of Prandtl, Reynolds and Marangoni numbers on the speed, shape, flow patterns and temperature distributions for solitary waves obtained from the regularized model. They also found that for small amplitude waves, the transport of heat by the flow has a stabilizing effect but promotes the instability of the large-amplitude waves when a recirculation zone is present. The bifurcation analysis of the first and second-order Benney equation for isothermal film flow was further carried out by Oron and Gottlieb [52]. The main result of their study is that, for smaller values of Reynolds number, the primary bifurcation of the first order Benney equation is supercritical and subcritical for larger values, while for the second order Benney equation, for any Reynolds number, the bifurcation is supercritical only. Later Samanta and Dandapat [53] studied the bifurcation analysis of first and second order Benney equation for viscoelastic fluid flowing down a vertical plane by using the method of multiple scales and they have found that the 
first order Benney equation gives both supercritical stable and subcritical unstable zones depending on the Reynolds number being smaller or greater than its critical value. They also obtained that the second order Benney equation exhibits only supercritical bifurcation and the supercritical stable region increases with increasing of Reynolds number.

Extensive review of the literature on the falling film instability down a heated vertical/inclined plane are found in Oron et al. [54], Velarde and Zeytounian [57], Nepomnyashchy et al. [56] and Colinet et al. [55].

Due to the technological applications, the effect of electric field on a thin liquid film produces a class of problems, which have got the attention of several researchers. Presence of the electric field introduces additional physical effects on the flow dynamics such as body force due to a current in conducting fluids and the Maxwell stress at the free interface. The electric field produces dispersion and hence linear stabilization of short wave modes. Schwarz and Melcher [58] examined the effects of an electric field on the linear stability of a sharp interface separating two non-conducting dielectric fluids of infinite extent. They also pointed out that the electric field introduces a polarization force which tends to deflect the interface from its initial flat position. The interaction of an electrostatic field on the film flowing in an inclined plane was studied by Kim et al. [59]. They obtained that, in the thin film limit, the effect of the electric field occurred in the wave evolution as an external pressure distribution that causes the destabilizing effect on the film. It is very interesting to note that, they provide a discussion on the application of their results to a proposed electrostatic liquid film space radiator. Further Gonzalez and Castellanos [60] investigated the nonlinear stability of a perfectly conducting film flowing down an inclined plane with linear temperature variation in the presence of a normal electric field. They derived a nonlinear evolution equation with a Hilbert transform type of term within the limit of small Reynolds number and predicted the destabilizing effect of the electric field in the finite amplitude regime. Later Dandapat and Mukhopadhyay [61] extended the study of Gonzalez and Castellanos within the regime of large Reynolds number and confirmed the existence of supercritical stable and subcritical unstable zones and also determined a critical value of the electric parameters below which the flow remains stable always.

Mukhopadhyay and Mukhopadhyay [62] investigated the stability of conducting viscous film flowing down an inclined plane with linear temperature variation in the presence of a uniform normal electric field. They found that both the Marangoni and electric Weber numbers have a qualitatively same influence on the stability characteristics but the effect of magnetic number is much stronger as compared to the effects of the electric Weber number.

The flow of the electrically conducting liquid film has several practical applications in different cooling systems, nuclear energy equipment and in other technological applications, e.g. in laser cutting process, where the surface waves are undesirable at the molten interface. Applying a magnetic field to counteract the inertia force, the instability could be prevented to maintain the smooth flow. This is more desirable since the electromagnetic field has no mechanical contact with the fluid flow.

The literature of MHD film flow down an inclined/vertical plane is sparse. In presence of a transverse uniform magnetic field, linear stability analysis for the electrically conducting liquid film flowing down an inclined plane was first investigated by Hsieh [63] and later followed by Ladikov [64], Sarma and Lu [65], Rai and Gupta [66]. It was found that the MHD coupling increases the stability of liquid film and can serve as a powerful remedy in suppressing instability of the flow. Gordeev and Murzenko [67] investigated wave flows of a conducting viscous fluid film over a horizontal plane in transverse constant magnetic and electric fields. They have considered an induction-free approximation to derive the evolution equation and discovered that the flow suffers from instability at a critical (sufficiently large) value of external electric field without any connection to the Reynolds number. The propagation of weakly nonlinear waves over a flow of an electrically conducting viscous film flowing down an inclined plane under the electromagnetic field by the long-wave expansion method was studied by Korsunsky [68]. He mainly performed the linear stability analysis of the evolution equation. He found that the magnetic field provides a stabilizing effect on the film flow, while, the electrical field destabilizes it. Further Dandapat and Mukhopadhyay [69] have studied the same problem as Korsunsky [68] and they performed both the linear and nonlinear stability analysis within the regime of small Reynolds number. Mukhopadhyay and Mukhopadhyay [70] investigated the stability of conducting liquid film flowing down an inclined plane at moderate Reynolds number in the presence of a constant electromagnetic field. They found that the magnetic field stabilizes the flow but the electric field stabilizes a disturbance of the flow depending on its orientation with the flow.

Another set of problems concern the effect of rotation on the thin liquid falling film. The hydrodynamic instability of a thin film flowing down a rotating inclined plane was first investigated by Ruiz-Chavarria and Davalos-Orozco [71]. They considered that the fluid is flowing down a rotating inclined plane close to the axis of rotation in order to neglect the centrifugal force. A stabilizing effect of the Coriolis force was found in their study and the linear instability of the thin film was described in the limit of small wave numbers and small Reynolds numbers. Later, Busse and Davalos-Orozco [72] studied the same problem by considering small wave number and small rotation approximation in such a way that both the forces, centrifugal and the Coriolis, have equal importance in the instability phenomena in the investigated region. They incorporated the effects of the centrifugal force together with the effects of the gravity and the Coriolis force in their study. They found a critical rate of rotation below which the film flowing down a rotating inclined plane is more stable than the flow in the horizontal rotating plane, while above this rate of rotation the situation is reversed.

Mukhopadhyay and Mukhopadhyay [73] studied the stability of a thin viscous liquid film flowing down a rotating nonuniform heated inclined plane and found that the Coriolis force is dominant for very small rotation while for relatively large rotation the centrifugal force is dominant.

Mukhopadhyay et al. [74] showed the impact of Biot number (Bi) which describes heat transfer at the free surface on instability mechanism. Using the long-wave expansion method, a generalized nonlinear evolution equation of Benney type was derived for the development of the free surface. They used a normal mode approach and the method of multiple scales to obtain the linear and weakly nonlinear stability solution for the film flow. The linear stability analysis of the evolution equation shows that the Biot number plays a double role; for $B i<1$, it gives destabilizing effect but for $B i>1$, it produces stabilization. At $B i=1$, the instability is maximum. The weakly nonlinear study reveals that the impact of Marangoni number $M r$ is very strong on the bifurcation scenario even for a slight variation. Recently Mukhopadhyay and Chattopadhyay [75] investigated interfacial instability of a thin liquid film flowing down an inclined plane where they considered the linear variation of fluid properties such as density, dynamical viscosity, surface tension, and thermal diffusivity, for the small variation of temperature. Using long-wave expansion method and considering order analysis especially for very small Biot number, they obtained a single surface equation in terms of the free surface $h(x, t)$. They discussed different stability zones and threshold amplitude in the subcritical as well as supercritical zones for the variation of the parameters for density, viscosity and surface tension, measuring the rate of change with respect to temperature and also showed the variation of nonlinear wave speed for the same variation. 
Study of the falling film instability draws the attention of several researchers for mainly two reasons. First, for its complexity of involving a free surface, which appealed to the applied mathematics community to formulate the classical flow instabilities, such as Tollmein-Schlicting, Kelvin-Helmholtz, Couette, Rayleigh, Poiseuille etc. Second, and the most important reason is its wide application in the practical field of industry and engineering. Experimental results have shown that presence of the waves on a liquid film surface makes a massive change on the coating surface because waves substantially enhance the heat and mass transfer rates. It is important in the other coating processes to suppress the surface waves on the fluid interface because the surface wave on a coating layer is highly undesirable as it decreases the glossy texture of the finished product. In many industrial and technological applications like microfabrication processes, coating or microelectromechanical systems etc., the temperature gradients are applied to control the flow of the liquid in order to impose uniform thickness of the liquid layers upon the solid surface. As surface tension is a temperature dependent property of the liquid, in the presence of the temperature gradient the free surface experiences the surface tension gradient or Marangoni stress and such stresses can generate the thermocapillary flows and interfacial instabilities. Thus any small variation in the temperature could lead to the growth of the instabilities that can disrupt the entire coating layer.

\section{Hydrodynamic Stability}

In fluid dynamics, hydrodynamic stability is the field which analyses the stability and the onset of the instability in fluid flows. The study of hydrodynamic stability aims to find out if a given flow is stable or unstable: if a physical state withstands a disturbance and it still returns to its original state after suffering all possible modes of disturbances, then it is in a stable state; otherwise, we can say that it is in unstable state. The foundations of hydrodynamic stability, both theoretical and experimental, were laid notably by Kelvin, Helmholtz, Rayleigh, and Reynolds during the nineteenth century. These foundations gave many useful tools to study hydrodynamic stability. The principle of the hydrodynamical stability considers a hydrodynamic system, in accordance with the equations governing it, is in a stationary state in which none of the variables describing the flow are dependent of time. Let $X_{1}, X_{2}, \ldots, X_{j}$ be a set of parameters which defines the system. These parameters will be geometrical parameters, that is parameters characterizing the velocity field, the angle of inclination for a flow on an inclined plane or the magnitudes of the different forces acting on the system, such as gravitational force, pressure gradients, temperature gradients, Coriolis force, magnetic fields, centrifugal force etc.

While considering the stability of such a system with the given set of parameters $X_{1}, X_{2}, \ldots, X_{j}$ we essentially try to determine the reaction of the system to small disturbances. Then the question arises whether the system which is disturbed will come back to its original state or whether moving further from the steady state away will never return to its original configuration. In the former case, the system is said to be in a stable state and in the latter case, it is said to be in an unstable state. More rigorously, a system is said to be in an unstable state if it is unstable with respect to a special mode of disturbances. On the other hand, a system is in a stable state if it is stable in every possible mode of disturbances. Marginal stability or Neutral stability is the locus in the parameter space $X_{1}, X_{2}, \ldots, X_{j}$ which separates the stable/unstable states. The locus of the marginal/neutral states in the $\left(X_{1}, X_{2}, \ldots, X_{j}\right)$ space can be defined by an equation of the form

$$
\sum\left(X_{1}, X_{2}, \ldots, X_{j}\right)=0 .
$$

The determination of this locus is one of the prime objects of the hydrodynamic stability.

In determining the stability of the hydrodynamical system, one particular parameter is chosen as a variable, keeping all the other parameters as constant. Now continuously varying that parameter, when the system changes from stable to unstable state for a certain critical value, we may say that, instability sets in for that critical value of the parameter when all the other parameters have their preassigned values.

States of neutral stability generally may be of two kinds; first the amplitude of the small disturbance may dampen/grow periodically; second, it may dampen/grow non-periodically. The core concern of the researcher is not the latter but the former case, where the transition from the stable to unstable state occurs via a marginal state exhibiting oscillatory motions with a certain definite characteristic frequency. Linearly unstable solutions cannot be realized. Linear theory predicts that flows diverge exponentially from unstable solutions. But as the difference becomes finite, the nonlinear effects can no longer be neglected. Nonlinearities usually cause a saturation of the exponential divergence and the flow evolves to a new steady, periodic or non-periodic flow. Typically, the resulting flow is spatially and/or temporally more complicated than the unstable solution. Nonlinear techniques have to be used to describe the resulting flows. One such simplification is provided by a weakly nonlinear analysis, which goes one step beyond linear stability analysis. In this analysis, the nonlinear terms are neglected assuming the perturbations to be small up to compared the leading order terms in an asymptotic expansion. Nonlinearities make their appearance at higher orders and may cause saturation of the exponential growth predicted by linear analysis for unstable flows. This analysis is only valid near the linear stability boundary and when the new flow is not very different from the unstable solution. Nevertheless, it addresses the question of what happens to an unstable flow.

\subsection{Linear stability analysis in terms of normal modes}

The starting point of investigation of hydrodynamic stability is the eigenvalue analysis, which proceeds in two stages:

1. Linearization about the laminar solution and

2. Search for unstable eigenvalues of the linearized problem.

An unstable eigenvalue is an eigenvalue in the complex upper half plane, corresponding to an eigenmode of the linearized problem which grows exponentially as a function of time $t$. A flow will be unstable if there exists such a growing eigenmode. Such modes of instability depend on the geometry (inclination angle of the inclined plane along which the film flows), the Reynolds number and other parameters. If the amplitude of the disturbance grows exponentially then at some point the nonlinear processes will become very important.

A widely used technique in the Hydrodynamic Stability Theory is the Linear Stability Analysis. In this analysis, at first, the evolution equations for perturbations about a steady solution are derived. The perturbation evolution equations are nonlinear, which makes them difficult to solve. Based on the assumption that the perturbations are infinitesimally small nonlinearity is neglected in this analysis. The means of infinitesimal disturbance is a disturbance in which its amplitude is small compared to any length scale such as its wave length This makes the evolution equation linear and is much easier to solve by superposition of the evolution operator. If all the modes of the 
linear operator eventually decay, any small perturbation will also degenerate and then the flow will return to the known steady solution. But stability will be lost when a single mode keeps growing with time. Thus the main task of this analysis is to determine whether any mode of the evolution operator shows persistent growth. In linear stability analysis, in order to investigate the reaction of the system to every possible mode of disturbances, we assume a very simple form for the disturbances, namely, Normal modes or Travelling waves $\eta=\eta_{0} \exp i\left(k_{x} x+k_{y} y-\omega t\right)+$ c.c. where, $\eta_{0}$ is the amplitude of the disturbance which is arbitrary but small, $k=\sqrt{k_{x}^{2}+k_{y}^{2}}=\frac{2 \pi}{\lambda}$ (where $\lambda$ is the wave length of the disturbance) is the wave number, which is real and $\omega=\omega_{r}+i \omega_{i}$ is the complex frequency and c.c. stands for complex conjugate. Here the growth is assumed to be exponential, which is the strongest possible disturbance and is observed in nature. Since $\exp (-i \omega t)=\exp \left(-i \omega_{r} t\right) \exp \left(\omega_{i} t\right)$, the disturbance will be unstable as time grows, if its imaginary part $\omega_{i}>0$ and will be stable if $\omega_{i}<$ 0 . The equation $\omega_{i}=0$ gives the neutral stability curve in the $(R e, k)$ plane (where $R e$ represents the Reynolds number), which separates the stable and unstable region.

The intersection of the two neutral curves determines the minimum $R e$ at which the instability sets in. This value is known as the critical Reynolds number $R e_{c}$, below of which the flow is always stable to infinitesimal disturbances. The flow may become unstable for $R e<R e_{c}$ under finite amplitude disturbances. This type of instability is known as subcritical instability. On the other hand, flow may become stable for $R e>R e_{c}$ under finite amplitude disturbances, known as supercritical stability. Also $c_{r}=\frac{\omega_{r}}{k}$ gives the linear phase speed of the wave propagation.

An important thing now arises from the above discussion is that, we can use a complex representation for a real disturbance, because of the fact that, although the disturbance is taken complex, to make it a real number we use the c.c. part. If $\eta=\eta_{0} \exp i\left(k_{x} x+k_{y} y-\omega t\right)+\eta_{0} \exp -$ $i\left(k_{x} x+k_{y} y-\omega t\right)$ is substituted in the evolution equation, we get twice as many terms. One set has $\eta_{0} \exp i\left(k_{x} x+k_{y} y-\omega t\right)$ as a factor and other set have $\eta_{0} \exp -i\left(k_{x} x+k_{y} y-\omega t\right)$ as a factor. For the expression to be always true for any $x, y$ and $t$ each of these sets separately must be equal to 0 .

\subsection{Weakly nonlinear stability analysis by the method of multiple scales}

Multiple scales, or more precisely, scales of different orders arise in many of the physical problems as different physical effects usually manifest themselves over a different length and time scales. So, it has wide applicability to the nonlinear wave propagation that involves physical phenomena, which occur in relation to various scales. Sturrock [76] first introduced the method of multiple scales for an investigation of nonlinear effects in the electron plasmas. The weakly nonlinear theory of hydrodynamic stability, first proposed by J. T. Stuart [77], became popular in the seventies when Benney [8] successfully developed this method for the nonlinear wave propagation of a thin film flowing down an inclined plane. A deep description of this method has been given by L. Debnath [78]. Excerpts of this are as follows. Let

$$
L\left(\frac{\partial}{\partial t}, \frac{\partial}{\partial x}, \lambda\right) u(x, t)=N\left(\frac{\partial}{\partial t}, \frac{\partial}{\partial x}, \mu\right) u^{2}(x, t) \quad x \in R, t>0
$$

is a nonlinear partial differential equation, where $L$ and $N$ are the differential operators and $\lambda, \mu$ are the fixed parameters. We introduce the sets of independent variables $x_{0}, x_{1}, \ldots, x_{m}$ and $t_{0}, t_{1}, \ldots, t_{m}$ defined by $x_{n}=\varepsilon^{n} x$ and $t_{n}=\varepsilon^{n} t$, where $\varepsilon$ is a small parameter characterizing the smallness of the associated terms. Consequently, the dependent variable $u(x, t)$ can be regarded as a function of these new variables, so that $u(x, t)=u\left(x_{0}, x_{1}, \ldots, x_{m}, t_{0}, t_{1}, \ldots, t_{m}\right)$.

Since the method is called the derivative expansion method, it is appropriate to introduce the expansions of the derivative operators

$$
\frac{\partial}{\partial x}=\sum_{n=0}^{m} \varepsilon^{n} \frac{\partial}{\partial x_{n}}
$$

and

$$
\frac{\partial}{\partial t}=\sum_{n=0}^{m} \varepsilon^{n} \frac{\partial}{\partial t_{n}}
$$

Using these expansions in (3.2) leads to the following results

$$
\begin{aligned}
& L\left(\frac{\partial}{\partial t}, \frac{\partial}{\partial x}, \lambda\right)=\sum_{n=0}^{m} \varepsilon^{n} L_{n}\left(\frac{\partial}{\partial t_{0}}, \frac{\partial}{\partial t_{1}}, \ldots, \frac{\partial}{\partial t_{m}}, \frac{\partial}{\partial x_{0}}, \frac{\partial}{\partial x_{1}}, \ldots, \frac{\partial}{\partial x_{m}}, \lambda\right)+O\left(\varepsilon^{m+1}\right), \\
& N\left(\frac{\partial}{\partial t}, \frac{\partial}{\partial x}, \mu\right)=\sum_{n=0}^{m} \varepsilon^{n} N_{n}\left(\frac{\partial}{\partial t_{0}}, \frac{\partial}{\partial t_{1}}, \ldots, \frac{\partial}{\partial t_{m}}, \frac{\partial}{\partial x_{0}}, \frac{\partial}{\partial x_{1}}, \ldots, \frac{\partial}{\partial x_{m}}, \mu\right)+O\left(\varepsilon^{m+1}\right) .
\end{aligned}
$$

We also assume that $u(x, t)$ has the asymptotic representation

$$
u\left(x_{0}, x_{1}, \ldots, x_{m}, t_{0}, t_{1}, \ldots, t_{m}\right)=\sum_{n=0}^{m} \varepsilon^{n} u_{n}\left(x_{0}, x_{1}, \ldots, x_{m}, t_{0}, t_{1}, \ldots, t_{m}\right)+O\left(\varepsilon^{m+1}\right) .
$$

In general, $u(x, t)$ can be expanded in terms of another small parameter $\delta$ which measures the degree of nonlinearity of the wave field. It is assumed that the new parameter is related to $\varepsilon$. However, to keep it simple, $u(x, t)$ has been expanded in powers of $\varepsilon$.

We substitute (3.5)-(3.7) in the equation (3.2) and then equate the coefficients of the like powers of $\varepsilon$ to obtain a system of perturbation equations from which it is possible to determine the functions $u_{n}$ successively. The underlying assumption is that each perturbed quantity $u_{n}$ must be nonsecular (bounded) at each stage of the perturbation process and thus, the method of the multiple scales can be applied effectively to a general dispersive wave system with or without small dissipation. 


\section{Formulation of the problem}

Consider a viscous, two-dimensional incompressible thin film flowing under the action of gravity along an inclined plane of inclination $\theta\left(0 \leq \theta \leq \frac{\pi}{2}\right)$ with the horizon. If the plane is vertical then $\theta=\frac{\pi}{2}$. Through $\theta$, the geometry of the flow is incorporated as a parameter. In the problem formulation, the liquid flowing has constant dynamical viscosity and density denoted by $\mu$ and $\rho$ respectively. The free surface of the liquid is bounded by an ambient air of constant atmospheric pressure $p_{a}$. The frame of reference is to be chosen in such a way that the $x$-axis is along the plane and $z$-axis perpendicular to the plane, and their intersection is the origin, which is a fixed point on the inclined/vertical plane. The dynamics of a falling liquid film is fairly well described as a free surface problem of two-dimensional single phase Navier-Stokes equation (momentum conservation) supplemented by continuity equation (mass conservation) and pertinent boundary conditions.

\section{Governing equations}

With respect to the chosen frame of reference, the governing equations in vector form are represented by

$$
\begin{aligned}
\rho\left[\frac{\partial \mathbf{V}}{\partial t}+(\mathbf{V} . \nabla) \mathbf{V}\right] & =\rho \mathbf{g}-\nabla p+\mu \nabla^{2} \mathbf{V} \\
\nabla . \mathbf{V} & =0
\end{aligned}
$$

where $\nabla=\left(\frac{\partial}{\partial x}, 0, \frac{\partial}{\partial z}\right)$ is the del operator, $\mathbf{V}=(u, 0, v)$ is the velocity vector, $\mathbf{g}=(g \sin \theta, 0,-g \cos \theta)$ is the gravitational acceleration and $p$ denotes the pressure of the liquid. To derive the above equation it is assumed that there is no influence of the air above the free surface.

\section{Boundary conditions}

The boundary conditions along the plane $z=0$ is the no-slip condition (i.e. fluid particles adhere to the plane do not move) which gives

$$
\mathbf{V}=0 \text {. }
$$

Also the free surface $z=h(x, t)$ which is in contact with the surrounding air, experiences two types of boundary conditions:

\section{(i) Kinematic condition}

It states that the liquid particles on the free surface must remain attached, which gives,

$$
\partial_{t} h+\mathbf{V} \cdot \nabla(h-z)=0 .
$$

(ii) Balance of normal and tangential stresses

The normal stress undergoes a jump across the interface with curvature of the free surface due to capillary force, which gives,

$$
[[\mathbf{n} \cdot \tau . \mathbf{n}]]-[[p]]=-\sigma_{0} \nabla . \mathbf{n}
$$

also the continuity of the shear stress gives,

$$
[[\mathbf{n} \cdot \tau . \mathbf{t}]]=0
$$

where, [[\#]] denotes a jump in the quantity as one moves across the interface from the liquid to vacuum region. Here $\mathbf{n}$ and $\mathbf{t}$ are the normal (outward pointing) and tangent vectors to the interface respectively, $\sigma_{0}$ is the constant surface tension of the liquid and the viscous stress tensor $\tau$ is given by $\tau=\left(\frac{\partial u_{i}}{\partial x j}+\frac{\partial u_{j}}{\partial x i}\right)$.

\section{Methodology}

The problem described above is well posed but it is almost difficult to find a unique solution by the use of existing mathematical tools. A steady state basic solution for the uniform parallel flow of the above problem can be obtained, which is popularly known as Nusselt's solution. However it is quite difficult to find a non-trivial solution of the problem. There are several reasons behind this. These are:

1. the governing mass and momentum conservation equations are coupled and the conservation equations are highly nonlinear,

2. the complexity of setting boundary conditions on the curvilinear interface,

3. presence of the different body force/forces, such as electromagnetic force, gravity, Coriolis force, centrifugal force etc.

Therefore, instead of finding the direct solution it would be wise to simplify the problem using certain type of approximations. There are two types of approximations available in the literature, which are widely used for the study of thin film flows. One approach is initiated by Benney [8] and Mei [79], and is known as 'long-wave expansion or perturbation expansion' technique The other approach, initiated by Kaptiza [5, 80] and Shkadov [9, 10], and is called 'integral-boundary layer or boundary-layer lubrication' method. Both the methods are discussed below. 


\section{(i) Long wave expansion method}

In this method the underlying assumption is that the average thickness of the fluid layer is much smaller in comparison with its characteristic length scale, whose order may be considered as the same of its wave length. Therefore the spatial gradients along the interface will be small. Now by expanding the dependent variables such as velocity, pressure etc. asymptotically in powers of the long-wave parameter $\varepsilon$ (the ratio of the average film thickness to the characteristic length scale) and substituting these in the governing equations together with the boundary conditions and then solving the leading order equations, we get the zeroth order solutions of the dependent variables. Considering the first order approximation of the governing equations and boundary conditions and using the solution of the zeroth order equations, we get the first order solutions of the dependent variables. Repeating this process, other leading order corrections of the dependent variables can be obtained. Finally substituting these corrected solutions in the kinematic equation we get a surface equation of the form

$$
\partial_{t} h=f\left(h^{n}, \partial_{x^{m}} h\right)
$$

which involves different algebraic powers $n$ and differentiation of orders $m$ of the film height $h(x, t)$. It is to be noted here that the long-wave expansion method becomes invalid when the asymptotic expansion of at least one dependent variable in terms of $\varepsilon$ diverges. In deriving long-wave equation it is assumed that the Reynolds number is very small and is of order unity $[O(1)]$. In this method the surface equation is valid only at a small vicinity of the critical Reynolds number, which is a serious limitation in the range of validity of the long-wave equation.

\section{(ii) Integral-boundary-layer method}

This method is a direct extension of the Karman-Polhausen integral-boundary layer theory. In this method initially the full Navier-Stokes equation is simplified by using the boundary-layer approximation and the pressure term is eliminated but the inertia term is left intact. Though the boundary layer approximation simplifies the Navier-Stokes equation considerably by dropping several terms, the resulting equation is still difficult to handle as this does not represent the so called surface equation. In the second phase, integrating the boundary layer equations across the film thickness by assuming a specific shape (parabolic or semi-parabolic) of the velocity profile for the transient and non-uniform flow, one obtains a coupled set of two equations in terms of two measurable quantities the flow rate $q(x, t)=\int_{0}^{h(x, t)} u(x, t, z) d z$ and film thickness $h(x, t)$ of the form

$$
\begin{gathered}
h_{t}+q_{x}=0, \\
q_{t}+f\left(h^{m}, q^{n}, \partial_{t^{i} x^{j}} f(h, q)\right)=0
\end{gathered}
$$

with some additional approximations, such as quasi-stationary approximation. The above coupled equations can be combined into a single, second order surface equation for the film thickness $h(x, t)$. The results of the integral-boundary layer method is in good agreement with the available experimental/numerical solutions in the range of moderate Reynolds number. Thus the range of validity is extended by this method.

\section{Conclusion}

In this work we tried to include the important findings of several researchers on the topic of thin film flow instability. Our study reveals the following:

1. Thin film flow instability has wide range of applications. The study of thin film helps to solve the problems in the practical field of industry and engineering as well as to develop the theoretical knowledge about many types of flow instability.

2. Several authors investigated the thermocapillary or Marangoni instability in the thin liquid film which is extensively used in coating industry for uniform thickness of the liquid layers on the solid surface.

3. The presence of electric field in the thin film flow along inclined plane introduces additional physical effects on the flow dynamics such as body force due to a current in conducting fluids and the Maxwell stress at the free interfaces. This type of study is important due to its technological applications.

4. Studies of several researchers on the thin film flow in the presence of constant electromagnetic field are important as this is used to solve the problems of magnetic film coating.

5. Stability of film flowing down a rotating inclined plane with or without heating effect is another important class of problems useful to solve in the paper industry.

6. There is ample scope of research for the hydrodynamical study of thin film flow along an inclined/vertical plane with a view to solving the problems of different industrial and technological issues, especially in coating industry.

\section{Acknowledgement}

The authors are grateful to Prof. Sukriti Ghosal, Principal, M.U.C. Women's College, Burdwan, India for proof reading and to enhance the quality of text of the paper. The First author acknowledges his sincerest gratitude to his parents Dr. Kusal Chattopadhyay and Mandira Chattopadhyay for their constant inspiration and encouragement throughout this research.

\section{References}

[1] H. Schlichting, Boundary Layer Theory, McGraw-Hill Book Company Inc, New York., (1955), 308-337

[2] C.C. Lin, Hydrodynamic Stability, Cambridge University Press, New York (1955)

[3] D.Y. Hsieh, S.P. Ho, Wave and stability in fluids., World Scientific, Singapore (1994)

[4] P.G. Drazin, W.H. Reid, Hydrodynamic Stability, Cambridge University Press, Cambridge(1981)

[5] P.L. Kapitza, S.P. Kapitza, Wave flow of thin layers of a viscous fluid: III. Experimental study of undulatory flow conditions. Sov. Phys. J. Exp. Theor.Phys., 19 (1949), 105-120 
[6] T.B. Benjamin, Wave formation in laminar flow down an inclined plane. J. Fluid Mech. 2 (1957),554-574

[7] C.S. Yih, Stability of liquid flow down an inclined plane. Phys. of Fluids 6(3) (1963), 321-334

[8] D.J. Benny, Long waves on liquid films. J. Math. Phys. 45 (1966), 150-155

[9] V.Ya. Shkadov, Wave models in the flow of a thin layer of a viscous liquid under the action of gravity. Izv. Akad. Nauk SSSR, Mekh. Zhidk. Gaza 1 (1967), 43-50

[10] V.Ya. Shkadov, Theory of wave flow of a thin layer of a viscous liquid. Izv. Akad. NaukSSSR, Mekh. Zhidk. Gaza 2 (1968), 20-25

[11] G.I. Sivashinsky, Nonlinear analysis of hydrodynamic instability in laminar flame, I. - Derivation of the basic equation, Acta Astronautica 4 (1977), $1177-1206$

[12] A. Pumir, P. Manneville, Y. Pomeau, On solitary waves running down an inclined plane. J. Fluid Mech. 135 (1983), 27-50

[13] B. Gjevik, Occurrence of finite-amplitude surface waves on falling liquid films. Phys. Fluids. 13 (1970), 1918-1925

[14] S.P. Lin, Finite-amplitude stability of a parallel flow with a free surface. J. Fluid Mech. 36 (1969), 113-126

[15] C. Nakaya, Long waves on a thin fluid layer flowing down an inclined plane. Phys. Fluids. 18 (1975), 1407-1412

[16] S.P. Lin, Finite amplitude side-band stability of a viscous film. J. Fluid Mech. 63 (1974), 417- 429

[17] M.V.G. Krishna, S.P. Lin, Nonlinear stability of a viscous film with respect to three-dimensional side-band disturbances. Phys. Fluids 20 (1977),

[18] J. Liu, J.D. Paul, J.P. Gollub, Measurements of the primary instabilities of film flows. J.Fluid Mech. 250 (1993), 69-101

[19] J. Liu, J.P. Gollub, Onset of spatially chaotic waves on flowing films. Phys. Rev. Lett. 70(15) (1993), 2289-2292

[20] J. Liu, J.P. Gollub, Solitary wave dynamics of film flows. Phys. Fluids 6 (1994), 1702-1712

[21] S.V. Alekseenko, V.E. Nakoryakov, B.G. Pokusaev, Wave formation on a vertical falling liquid film. AIChE J. 31 (1985), 1446-1460

[22] H.C. Chang, Wave evolution on a falling film. Annu. Rev. Fluid Mech. 26 (1994), 103-136

[23] G.D. Fulford, The flow of liquid in thin films. Adv. Chem. Engg. 5 (1964), 151-236

[24] S.P. Lin, C.Y. Wang, Modeling Wavy film flows. In: Encyclopedia of Fluid Mechanics (ed. Cheremisinoff, N. P.) (1985), 931-951

[25] T.J. Hanratty, Interfacial instabilities caused by airflow over a thin liquid layer. In waves on Fluid Interfaces, Academic Press, London (1983), 221-259

[26] T.J. Hanratty, Separated flow modeling and interfacial transport phenomena. Appl. Sci.Res. 48 (1991), 353-390

[27] G.F. Hewitt, M.S. Hall-Taylor, Annular Two-Phase Flow. Pergamon Press, Oxford (1970)

[28] V.E. Nakaroyakov, B.G. Pokusaev, I.R. Schreiber, Wave Propagation in Gas-Liquid Media.Begell, New York (1992)

[29] J.R.A. Pearson, On convection cells induced by surface tension. J. Fluid Mech 4 (1958), 489- 500

[30] L.E. Scriven, C.V. Sternling, On cellular convection driven by surface tension gradients: effects of mean surface-tension and surface viscosity. J. Fluid Mech. 19 (1964), 321-340

[31] K.A. Smith, On convective instability induced by surface-tension gradients. J. Fluid Mech.14 (1966), 401-414

[32] S.G. Bankoff, Significant questions in thin liquid-film heat-transfer. ASME J. Heat Transfer 116 (1994), 10-16

[33] J.B. Burelbach, S.G. Bankoff, S.H. Davis, Nonlinear stability of evaporating/condensing liquid films. J. Fluid Mech. 195 (1988), 463-494

[34] D.A. Goussis, R.E. Kelly, Surface waves and thermocapillary instabilities in a liquid film flow. J. Fluid Mech. 223 (1991), 24-45

[35] S.W. Joo, S.H. Davis, S.G. Bankoff, Long-wave instabilities of heated falling films: two-dimensional theory of uniform layers. J. Fluid Mech. 230 (1991), 117-146

[36] P.G. Lopez, S.G. Bankoff, M.J. Miksis, Non-isothermal spreading of a thin liquid film on an inclined plane. J. Fluid Mech. 324 (1996), 261-286

[37] O.A. Kabov, I.V. Marchuk, V. Chupin, Thermal imaging study of the liquid film flowing on a vertical surface with local heat source. Russ. J. Engng. Thermophys. 6 (1996), 105-138

[38] O.A. Kabov, Formation of regular structures in a falling liquid film upon local heating. Thermophys. Aeromech. 5 (1998), 547-551

[39] B. Scheid, O.A. Kabov, C. Minetti, P. Colinet, J.C. Legros, Measurement of free surface deformation by the reflectance-Schlieren method. In Proc. 3rd. European Thermal Sciences conference, (ed. E. W. P. Hahne et al.), Heidelberg (2000)

[40] S. Kalliadasis, A. Kiyashko, E.A. Demekhin, Marangoni instability of a thin liquid film heated from below by a local heat source. J. Fluid Mech. 475 (2003), 377-408

[41] S. Kalliadasis, E.A. Demekhin, C. Ruyer-Quil, M.G. Velarde, Thermocapillary instability and wave formation on a film falling down a uniformly heated plane. J. Fluid Mech. 492 (2003),303-338

[42] E.A. Demekhin, V.Ya. Shkadov, Three-dimensional waves in a liquid flowing down a wall.Izv. Akad. Nauk SSSR, Mekh. Zhidk. Gaza 5 (1984), 21-27

[43] C. Ruyer-Quil, B. Scheid, S. Kalliadasis, M.G. Velarde, R.Kh. Zeytounian, Thermocapillary long waves in a liquid film flow. Part 1 Low-dimensional formulation. J . FluidMech. 538 (2005), 199-222

[44] P.M.J. Trevelyan, S. Kalliadasis, Wave dynamics on a thin-liquid film falling down a heated wall. J. Engineering Mathematics 50 (2004), 177-208

[45] S. Miladinova, S. Slavtchev, G. Lebon, J.C. Legros, Long-wave instabilities of non- uniform heated falling films. J. Fluid Mech. 453 (2002), 153-175

[46] S. Miladinova, D. Staykova, G. Lebon, B. Scheid, Effect of nonuniform wall heating on the three-dimensional secondary instability of falling films. Acta Mechanica 30 (2002), 1-13

[47] A. Mukhopadhyay, A. Mukhopadhyay, Nonlinear stability of viscous film flowing down an inclined plane with linear temperature variation. Journal of Physics D Applied Physics. 40(18) (2007), 5683-5690

[48] L.Y. Yeo, R.V. Craster, O.K. Matar, Marangoni instability of a thin liquid film resting on a locally heated horizontal wall. Physical Review E 67 (2003), $056315.1-056315.14$

[49] E.A. Demekhin, S. Kalliadasis, M.G. Velarde, Suppressing falling film instabilities by Marangoni forces. Phys. Fluids 18 (2006), 042111-1-16

[50] B. Scheid, C. Ruyer-Quil, U. Thiele, O.A. Kabov, J.C. Legros, P. Colinet, Validity domain of the Benney equation including Marangoni effect for closed and open flows. J. Fluid Mech. 527 (2004), 303-335

[51] B. Scheid, C. Ruyer-Quil, S. Kalliadasis, M.G. Velarde, R.Kh. Zeytounian, Thermocapillary long waves in a liquid film flow. Part 2. Linear stability and nonlinear waves. J.Fluid Mech. 538 (2005), 223-244

[52] A. Oron, O. Gottlieb, Subcritical and supercritical bifurcations of the first and second- order Benney equations. J. Engineering Mathematics 50 (2004), $121-140$

[53] B.S. Dandapat, A. Samanta, Bifurcation analysis of first and second order Benney equations for viscoelastic fluid flowing down a vertical plane. J.Phys. D: Appl. Phys. 41 (2008), 095501

[54] A. Oron, Nonlinear dynamics of thin evaporating liquid films subject to internal heat generation. In Fluid Dynamics at Interfaces, (ed. W. Shyy and R. Narayanan), Cambridge University Press (1999)

[55] P. Colinet, J.C. Legros, M.G. Velarde, Nonlinear dynamics of surface-tension-driven instabilities, Wiley VCH (2001)

[56] A.A. Nepomnyashchy, M.G. Velarde, P. Colinet, Interfacial phenomena and convection.Chapman and Hall (2002)

[57] M.G. Velarde, R.Kh. Zeytounian, Interfacial Phenomena and the Marangoni effect. Springer (2002)

[58] J.R. Melcher, W.J. Schwartz, Interfacial relaxation over stability in a tangential electric field. Phys. Fluids 11 (1968), 2604-2616

[59] H. Kim, S.G. Bankoff, M.J. Misksis, The effect of an electrostatic field on film flowing down an inclined plane. Phys. Fluids 4 (1992), 2117-2130

[60] A. Gonzalez, A. Castellanos, Nonlinear electrodynamic waves on film falling down an inclined plane. Phys. Rev. E 53 (1996), $3573-3578$

[61] A. Mukhopadhyay, B.S. Dandapat, Nonlinear stability of conducting viscous film flowing down an inclined plane at moderate Reynolds number in the presence of a uniform normal electric field. J. Phys. D: Appl. Phys. 38 (2005), 138-143

[62] A. Mukhopadhyay, A. Mukhopadhyay, Stability of conducting viscous film flowing down an inclined plane with linear temperature variation in the presence of a uniform normal electric field. Int. J. Heat and Mass Transfer. 52 (2009), 709-715

[63] D.Y. Hsieh, Stability of a conducting fluid flowing down an inclined plane in a magnetic field. Phys. Fluids 8 (1965), 1785-1791

[64] Yu.P. Ladikov, Flow stability of a conducting liquid flowing down an inclined plane in the presence of a magnetic field. Fluid Dynamics 8 (1966), 1-4

[65] P.C. Lu, G.S.R. Sarma, Magnetohydrodynamic gravity-capillary waves in a liquid film.Phys. Fluids 10 (1967), 2339-2344

[66] A.S. Gupta, L. Rai, Hydrodynamic stability of a liquid film flowing down an inclined conducting plane. J. Phys. Soc. Japan 24 (1968), 626-632

[67] Yu.N. Gordeev, V.V. Murzenko, Wave film flows of a conducting viscous fluid in the tangential magnetic field. Appl. Math. Theor.Phys. 3 (1990), 96-100

[68] S. Korsunsky, Long waves on a thin layer of conducting fluid flowing down an inclined plane in an electromagnetic field. Eur. J. Mech. B/Fluids 18 (1999), 295-313

[69] B.S. Dandapat, A. Mukhopadhyay, Finite amplitude long wave instability of a film of conducting fluid flowing down an inclined plane in presence of an electromagnetic field. Int. J.Appl. Mech. Engg. 8 (2003), 379-383

[70] A. Mukhopadhyay, A. Mukhopadhyay, Stability of conducting liquid film flowing down an inclined plane at moderate Reynolds number in the presence of a constant electromagnetic field. Int. J. Non-Linear Mechanics. 43(7) (2008), 632-642

[71] L.A. Davalos-Orozco, G. Ruiz-Chavarria, Hydrodynamic stability of a fluid layer flowing down a rotating inclined plane. Phys. Fluids A 4 (1992), 1651-1665 
[72] L.A. Davalos-Orozco, F.H. Busse, Instability of a thin film on a rotating horizontal or inclined plane. Physical Review E 65 (2002), 026312.1-026312.10

[73] A. Mukhopadhyay, A. Mukhopadhyay, Stability of a thin viscous fluid film flowing down a rotating non-uniformly heated inclined plane. Acta Mechanica 216(1) (2011), 225-242

[74] A. Mukhopadhyay, A. Mukhopadhyay, S. Mukhopadhyay, Instabilities of thin viscous film flowing down a uniformly heated inclined plane. J. of heat and mass transfer research $3(2015), 1-22$

[75] A. Mukhopadhyay, S. Chattopadhyay, Long wave instability of thin film flowing down an inclined plane with the linear variation of thermophysical properties for very small Biot number. Int J. of Non-Linear Mechanics 100 (2018), 20-29

[76] P.A. Sturrock, Non-linear effects in electron plasmas. A Royal Society journal 242 (1957), issue 1230

[77] J.T. Stuart, On the non-linear mechanics of wave disturbances in stable and unstable parallel flows Part 1. The basic behavior in plane Poiseuille flow. J. Fluid Mech. 9 (1960), 353-370

[78] L. Debnath, Nonlinear partial differential equations for scientists and engineers. Springer (2005)

[79] C.C. Mei, Nonlinear gravity waves in a thin sheet of viscous fluid. J. Math. Phys. 45 (1966), 266-288

[80] P.L. Kapitza, Wave flow of thin layers of a viscous fluid. Sov. Phys. J. Exp. Theor. Phys. 18 (1948), 3-28 